\title{
CONTOURLET-BASED FINGERPRINT ANTISPOOFING
}

\author{
Shankar Bhausaheb Nikam ${ }^{1}$ and Suneeta Agarwal ${ }^{2}$ \\ ${ }^{1}$ Department of Computer Engineering, \\ Government Polytechnic, Awasari, Pune \\ shankar_nikamerediffmail.com \\ ${ }^{2}$ Department of Computer Engineering, \\ Motilal Nehru National Institute of Technology, Allahabad \\ suneeta@mnnit.ac.in
}

\begin{abstract}
We propose an image-based method using Contourlet transform [5] to detect liveness in fingerprint biometric systems. We observe that real and spoof fingerprint images exhibit different textural characteristics. Wavelet transform although widely used for liveness detection is not the ideal one. Wavelets are not very effective in representing images containing lines and contours [5]. Recent Contourlet transform allows representing contours in a more efficient way than the wavelets [5]. Fingerprint is made of only contours of ridges; hence Contourlet transform is more suitable for fingerprint processing than the wavelets. Therefore, we use Contourlet energy and co-occurrence signatures to capture textural intricacies of images. After downsizing features with Plus $l$ - take away $r$ method, we test them on various classifiers: logistic regression, support vector machine and AdTree using our databases consisting of 185 real, 90 Fun-Doh (Play-Doh) and 150 Gummy fingerprint images. We then select the best classifier and use at as a base classifier to form an ensemble classifier obtained by fusing a stack of " $K$ " base classifiers using the "Majority Voting Rule" (i.e. bagging). Experimental results indicate that, the new liveness detection approach is very promising as it needs only one fingerprint and no extra hardware to detect vitality.
\end{abstract}

\section{KEYWORDS}

Bagging, Contourlet transform, Fingerprint, Liveness, Texture, Spoofing

\section{INTRODUCTION}

Recent studies have demonstrated that fingerprint biometric systems are vulnerable to spoofing [1]-[4]. Since these systems are extensively being used for authentication applications nowadays, it is imperative to enhance their security. This paper proposes an image-based method using recently introduced Contourlet transform to alleviate the problem of spoof fingerprint attacks. According to recent papers, wavelets are good in representing point discontinuities but are not suitable for representing smooth contours [5]. Wavelets can be used to represent point discontinuity in one dimension [5]. But, images in two dimensions have discontinuities along smooth contours [5]. So other ways of multiscale representation are required for images [5]. Do and Vetterli [5] proposed a new multiscale Contourlet transform to represent contours more efficiently than the wavelet transform. 
Fingerprint is made of only contours of ridges flowing in various directions; hence Contourlet transform is more suitable for fingerprint processing than the wavelets. Therefore, our method uses Contourlet transform to capture intrinsic textural differences in real and spoof fingerprints for liveness detection.

A simple visual analysis of real and spoof fingerprint images (see Figure 2) shows that, these images exhibit textural differences clearly, which can be characterized by Contourlet transformbased texture features. For example, ridge lines of real fingerprint images exhibit random gray level variations, hence have low gray level uniformity. Unlike real fingerprint images, spoof fingerprint images (Fun-Doh and Gummy) exhibit minute grey level variations, hence have high gray level uniformity. Moreover, we found that the skin properties of real fingers differ from the spoof fingerprints showing variation in various fingerprint texture characteristics (ridge width, ridge distribution, gray level pixel values distribution, ridge frequency, ridge discontinuities, etc.)

Figure 1 shows schematic of our Contourlet transform-based method to detect liveness. We apply Contourlet transform to decompose an image in various subbands of coefficients (details given in Section 3). We calculate Contourlet energy and co-occurrence signatures from Contourlet subbands to capture inherent textural intricacies of images. After dimension reduction with Plus $l$ - take away $r$ method [6], we test both Contourlet energy and co-occurrence signatures independently on various classifiers: logistic regression (LR) [7], support vector machine (SVM) [8], alternating decision tree (AdTree) [9] and an ensemble classifier [10]. We then combine energy and co-occurrence signatures to get a fused signature and test it on an ensemble classifier. Overall classification rate obtained with an energy signature ranges from $93.18 \%$ to $97.41 \%$. Similarly, overall classification rate obtained with co-occurrence signature ranges from $93.41 \%$ to $97.88 \%$. Fused signature is found to yield higher overall classification rate $98.59 \%$ relative to individual energy and co-occurrence signatures.

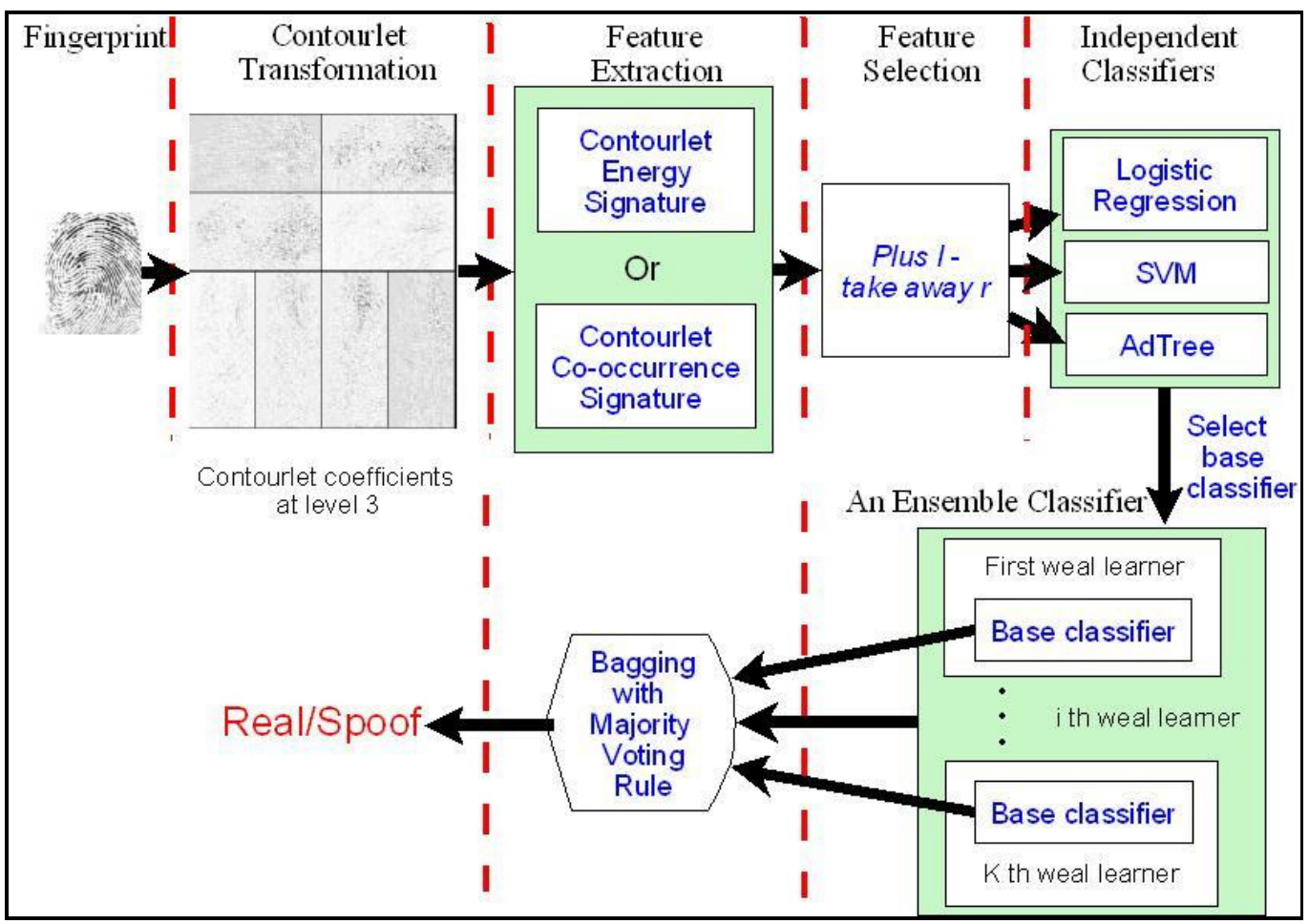

Figure 1. Schematic diagram of the proposed Contourlet-based liveness detection method. 


\section{RELATED WORKS}

Spoofing means fraudulent entry of an unauthorized person into a fingerprint biometric system by using a spoof (fake) fingerprint of an authorized person [1]-[4]. Liveness detection is one of the efficient countermeasures of anti-spoofing. Several liveness measures: temperature, odor, impedance and electrical conductivity of the skin, laser detection of 3-D finger surface and pulse, spectroscopy, skin perspiration, pulse oximetry, blood pulsation, E.C.G, skin elasticity, etc are proposed in the literature [4], [11], [12]. In wavelet-based liveness detection [13], surface coarseness is treated as a kind of Gaussian white noise added to the images. Wavelets are first used to denoise images and then the noise residue is estimated [13]. Researchers [13] used standard deviation of the noise residue to indicate the texture coarseness. Methods using perspiration need finger to be placed on a scanner surface for 2 seconds (or 5 seconds) to capture perspiration details; hence these methods are slow [14]. Some other aforementioned methods need extra hardware to capture physiological information for liveness detection [4]. Our proposed method alleviates these problems as it needs only one fingerprint and no extra hardware to detect liveness.

\section{PROPOSED APPROACH}

\subsection{Real, Fun-Doh and Gummy fingerprint databases}

Fingerprint images are acquired from 185 real (live), 90 Fun-Doh and 150 Gummy fingers using Secugen optical fingerprint scanner (Model- HFDU01). Casts and moulds of spoof fingers are made using different combinations of artificial materials. We use various materials: plumber's putty, dental impression materials, soft plastic, playing clay, RTV silicone, etc to create casts of the spoof fingerprints [15]. We use playing clay (e.g. Fun-Doh) and Fevi-gum to create moulds of "Fun-Doh" fingers and "Gummy" fingers, respectively [15]. We create spoof fingers for the same set of persons whose real fingerprints are in our database. Figure 2 shows parts of real, Fun-Doh and Gummy fingerprint images of one finger.

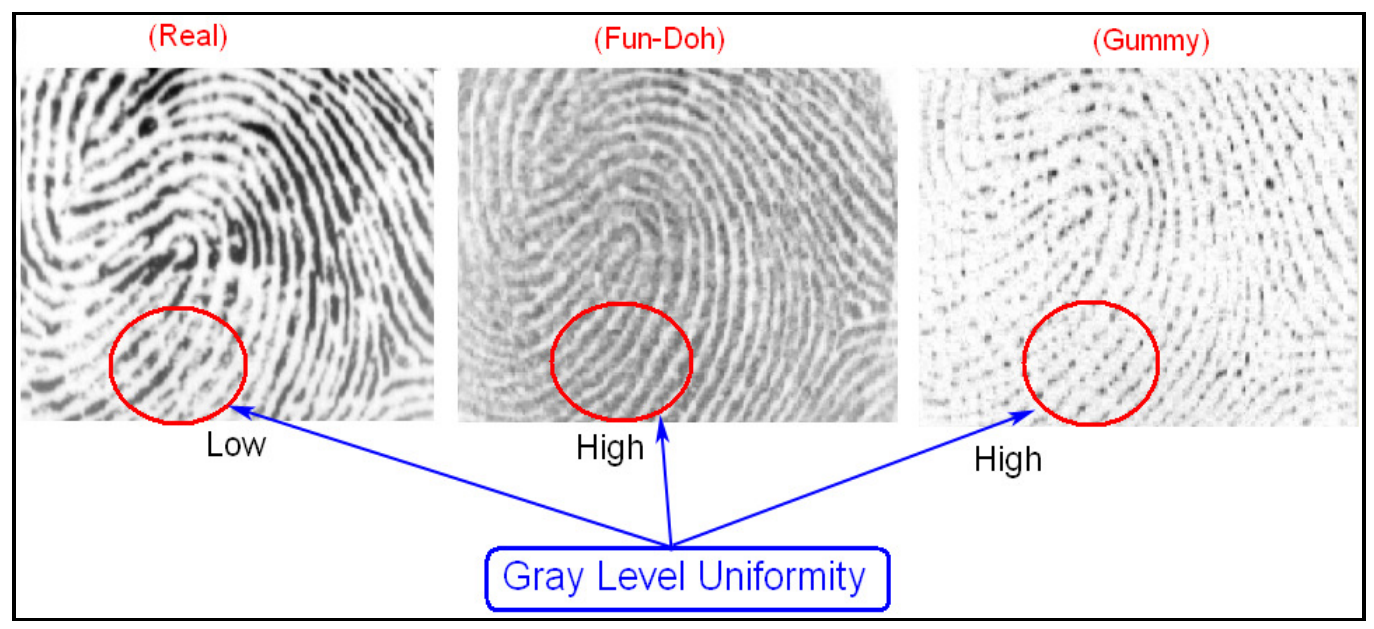

Figure 2. Parts of real, Fun-Doh and Gummy fingerprint images of one finger.

\subsection{Contourlet transform: a brief review}

Contourlet transform, also called pyramidal directional filter bank (PDFB), proposed by Do and Vetterli [5] efficiently represents contours and texture of an image. Contourlet transform consists 
of two steps: multiscale decomposition and the directional decomposition [5]. These steps expand an image using basic elements like contour segments, hence named the Contourlet [5]. Contourlet effectively capture the intrinsic contours in fingerprint images.

\subsubsection{Energy and co-occurrence signatures extraction}

We apply Contourlet transform at various pyramidal and directional levels to extract features [5]. We found that, features obtained with two pyramidal levels with one decomposition level at each pyramidal level yield the highest discriminating power (see Table 1). Hence, we decompose an image using two pyramidal levels with one decomposition level at each pyramidal level to get five subbands (one low pass and four directional band pass subbands) [5]. We do not use low pass subband because it does not contain high frequency texture information useful to detect liveness. We compute energy and mean deviation features [16] from each directional subband and then combine them to get Contourlet energy signature of the size 8 (4 directional subbands $\times 2$ features from each).

We also compute gray level co-occurrence matrix (GLCM) for each subband. The GLCM element $C(i, j, d, \theta)$ represent probability that pair of pixels, which are located with an intersample distance $d$ and a direction $\theta$, have a gray level $i$ and a gray level $j$. Thus, GLCM characterizes second order statistics of Contourlet coefficients. We compute GLCMs for various combinations of $d$ and $\theta$. Features computed from GLCM with $d=1$ and $\theta=45^{\circ}$ are found to provide best results (see Table 1). Hence, we use $d=1$ and $\theta=45^{\circ}$ to compute GLCM for each directional subband. We then compute 13 texture features from each GLCM: angular second moment, contrast, correlation, sum of squares, inverse difference moment, sum average, sum variance, sum entropy, entropy, difference variance, difference entropy, information measures of correlation (two features) [17] and concatenate them to get Contourlet co-occurrence signature of the size 52 ( 4 directional subbands $\times 13$ texture features from each).

\subsection{Plus $l$-take away $r$ method}

For downsizing features, we use Plus $l$ - take away $r$ method [6] which is a combination of Sequential Forward Selection (SFS) and Sequential Backward Selection (SBS) feature selection methods [6]. In Plus $l$ - take away $r$ method Sequential Forward Selection (SFS) is applied $l$ times which is then followed by $r$ steps of Sequential Backward Selection (SBS)[6]. Forward and backward selections are repeated until the required numbers of features are obtained [6]. Therefore, If $l>r$ it results in forward method and if $l<r$ it results in backward method [6].

\section{EXPERIMENTAL RESULTS}

\subsection{Classification results}

We test Contourlet energy and co-occurrence signatures independently on various classifiers. First LR classifier is chosen, as it is simple, fast and more accurate. It is a statistical model suitable for probabilistic binary classification [7]. SVM is a state-of-the-art classifier, so it is selected as a second classifier [8]. Third, an AdTree classifier is also used. An AdTree classifier is

a combination of decision trees with boosting. It is widely used for pattern classification problems [9].

10 fold (default) cross-validation results obtained with these classifiers are reported in Table 1. We observe that performances of both energy and co-occurrence signatures on all the three classifiers are promising. We found that for both energy and co-occurrence signatures, LR 
classifier provide best results (see table 1). Hence, we select LR classifier and use it as a base classifier (weak learner) to build an ensemble classifier (strong classifier). We use the "Bagging" algorithm [10] to fuse a stack of " $K$ " $(K=25)$ base classifiers (i.e. LR classifier) using the "Majority Voting Rule" [10] to get an ensemble classifier. We found that an ensemble classifier provides better results than the other classifiers. Next we fuse energy and co-occurrence signatures to get a fused signature and test it on an ensemble classifier. We found that fused signature enhances accuracy up to $98.59 \%$.

We also perform cross-validation testing of a fused signature on an ensemble classifier by varying the number of folds $(\mathrm{N})$. Moreover, we test a fused signature on an ensemble classifier by varying the value of "percentage data split" for training. In this testing option, a database is split in two parts. Certain percentage of a database is used for training and rest is held out for testing. Overall classification rates found with above testing methods are reported in Figure 3.

The experiments are performed on Pentium-4, $2.8 \mathrm{GHz}$ Processor with 2 GB RAM, running Windows XP. Time required for energy and co-occurrence signatures extraction is 0.0165 seconds and 0.968 seconds, respectively. Thus, energy signature is computationally efficient.

Table 1: Confusion matrices for 10-fold cross-validation; Features used: (1) Contourlet Energy Signature, (2) Contourlet Co-occurrence signature; No. of fingerprints used: Real-185, Fun-Doh-90, Gummy-150.

\begin{tabular}{|c|c|c|c|c|c|c|c|c|}
\hline & \multicolumn{4}{|c|}{ Contourlet Energy Signature } & \multicolumn{4}{|c|}{ Contourlet Co-occurrence Signature } \\
\hline & \multicolumn{2}{|c|}{ Assigned class } & \multirow{2}{*}{$\begin{array}{c}\text { Success } \\
(\%)\end{array}$} & \multirow{2}{*}{$\begin{array}{l}\text { Overall } \\
\text { class. rate } \\
\text { for } \\
\mathbf{a} \\
\text { classifier }\end{array}$} & \multicolumn{2}{|c|}{$\begin{array}{c}\text { Assigned } \\
\text { class }\end{array}$} & \multirow{2}{*}{$\begin{array}{c}\text { Success } \\
(\%)\end{array}$} & \multirow{2}{*}{$\begin{array}{c}\text { Overall } \\
\text { class. rate } \\
\text { for } \\
\text { a classifier }\end{array}$} \\
\hline $\begin{array}{l}\text { True } \\
\text { class }\end{array}$ & Real & Spoof & & & Real & Spoof & & \\
\hline \multicolumn{9}{|c|}{ Logistic Regression (LR) Classifier } \\
\hline Real & 179 & 06 & 96.76 & \multirow{3}{*}{96.47} & 183 & 02 & 98.92 & \multirow{3}{*}{97.65} \\
\hline Fun-Doh & 04 & 86 & 95.56 & & 05 & 85 & 94.44 & \\
\hline Gummy & 05 & 145 & 96.67 & & 03 & 147 & 98.00 & \\
\hline \multicolumn{9}{|c|}{ Support Vector Machine (SVM) Classifier } \\
\hline Real & 176 & 09 & 95.14 & \multirow{3}{*}{93.88} & 176 & 09 & 95.14 & \multirow{3}{*}{93.41} \\
\hline Fun-Doh & 07 & 83 & 92.22 & & 08 & 82 & 91.11 & \\
\hline Gummy & 10 & 140 & 93.33 & & 11 & 139 & 92.67 & \\
\hline \multicolumn{9}{|c|}{ Alternating Decision Tree (AdTree) Classifier } \\
\hline Real & 174 & 11 & 94.05 & \multirow{3}{*}{93.18} & 179 & 06 & 96.76 & \multirow{3}{*}{95.76} \\
\hline Fun-Doh & 07 & 83 & 92.22 & & 05 & 85 & 94.44 & \\
\hline Gummy & 11 & 139 & 92.67 & & 07 & 143 & 95.33 & \\
\hline \multicolumn{9}{|c|}{ Ensemble Classifier } \\
\hline Real & 182 & 03 & 98.38 & \multirow{3}{*}{97.41} & 183 & 02 & 98.92 & \multirow{3}{*}{97.88} \\
\hline Fun-Doh & 05 & 85 & 94.44 & & 03 & 87 & 96.67 & \\
\hline Gummy & 03 & 147 & 98.00 & & 04 & 146 & 97.33 & \\
\hline
\end{tabular}




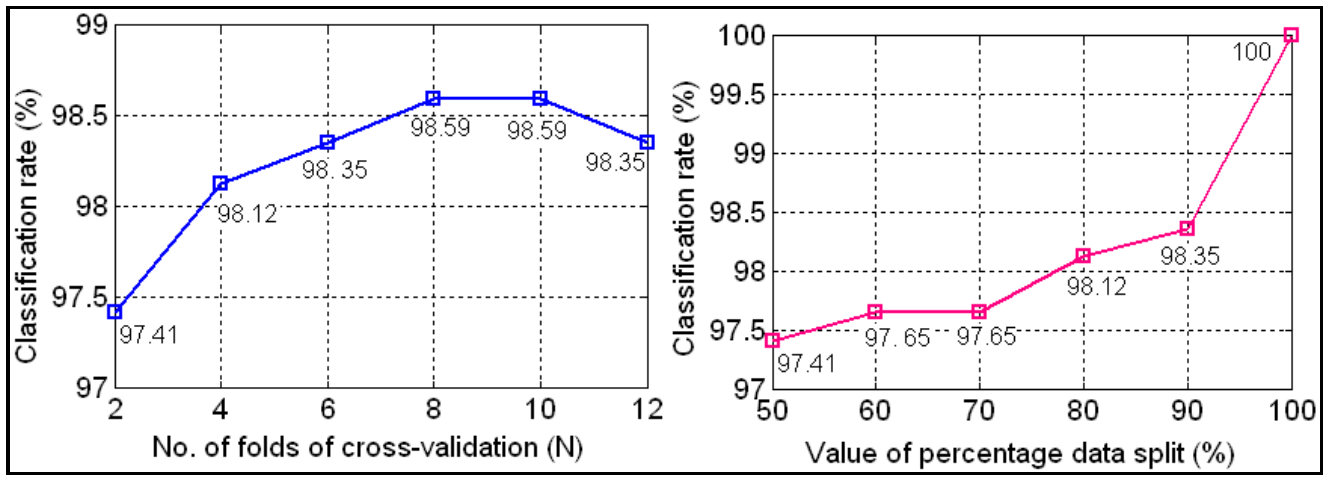

Figure 3. Overall classification rates achieved for real, Fun-Doh and Gummy fingerprint images classification by varying: (a) number of folds of cross-validation (N), (b) value of percentage data split; features: fused signature; classifier: an ensemble classifier.

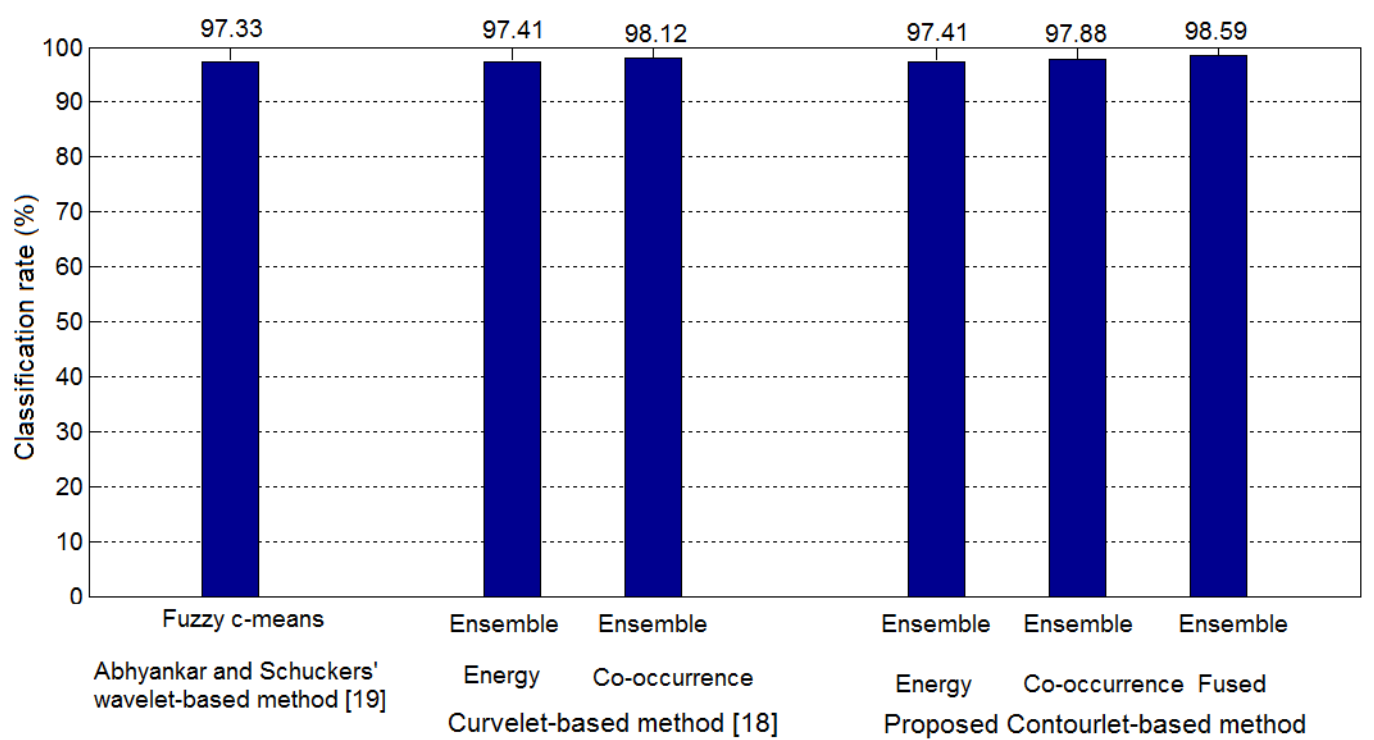

Figure 4. Comparison with Curvelet-based method [18] and Abhyankar and Schuckers' method [19] for real and spoof fingerprint classification.

\subsection{Comparison with related work}

We select two liveness detection methods for comparison. First is our curvelet-based method [18] and second one is Abhyankar and Schuckers' single-image-based method [19] using wavelets and fuzzy-c-means classifier. We implement the method in [19] and test it on our databases consisting of both real and spoof fingerprint images. We found that the method [19] provides $97.33 \%$ accuracy. We found that (see Figure 4) our proposed features (energy, co-occurrence and fused) using recent Contourlet transform clearly outperform wavelet-based method [19]. However, curvelet-based co-occurrence features [18] yield slightly more accuracy than the Contourlet-based co-occurrence features. The performances of energy features for both the tools: Contourlet and Curvelet are found to be the same. 
Computer Science \& Information Technology (CS \& IT)

\section{CONCLUSIONS AND Future Directions}

We present a new Contourlet-based method using textural features (energy and co-occurrence signatures) to detect liveness in fingerprint biometric systems. After dimension reduction using Plus $l$ - take away $r$ method, we analyze features using various classifiers: logistic regression, SVM, ADTree and an ensemble classifier formed using the "Bagging" ensemble method. Our fused signature (fusion of energy and co-occurrence signatures) is found to yield highest accuracy (98.59\%). We found that our Contourlet-based method is more efficient than the related waveletbased method [19]. Also, our method needs only one image to detect liveness; hence it reduces the cost of a biometric system as no additional hardware is required. Following are some future directions:

1. One can work on efficient ways of modeling Contourlet coefficients to extract more distinctive texture information.

2. Selection of best GLCM parameters using optimization tools such as genetic algorithms is also possible.

\section{REFERENCES}

[1] N. K. Ratha, (2001) "Enhancing security and privacy in biometrics-based authentication systems", IBM Systems Journal, Vol. 40, No. 3, pp. 614-634.

[2] T. V. D. Putte, J. Keuning, (2000) "Biometrical fingerprint recognition: don't get your fingers burned", Proceedings of $4^{\text {th }}$ Working Conference on Smart Card Research Advanced Applications, pp. 289-303.

[3] T. Matsumoto, H. Matsumoto, K. Yamada, and S. Hoshino, (2002) "Impact of artificial 'Gummy' fingers on fingerprint systems", Proceedings of SPIE, Optical Security \& Counterfeit Deterrence IV, Vol. 4677, pp. 275-289.

[4] S. A. C. Schuckers, (2002) "Spoofing and anti-spoofing measures", Information Security Technical Report, Vol. 7, No. 4, pp. 56-62.

[5] M. N. Do, M. Vetterli, (2005) "The contourlet transform: an efficient directional multiresolution image representation”, IEEE Transactions on Image Processing, Vol. 14, No. 12, pp. 2091-2106.

[6] P. Pudil, J. Novovicova, J. Kittler, (1994) "Floating search methods in feature selection", Pattern Recognition Letters, Vol. 15, No. 11, pp. 1119-1125.

[7] S. L. Cessie, J. C. Houwelingen, (1992) "Ridge estimators in logistic regression", Applied statistics Vol. 41, No. 1, pp. 191-201.

[8] LIBSVM - A library for support vector machine (SVM) classifier $<$ http://www.csie.ntu.edu.tw/ cjlin/libsvm/>

[9] Y. Freund, L. Mason, (1999) "The alternating decision tree algorithm", Proceedings of the $16^{\text {th }}$ International Conference on Machine Learning, Bled, Slovenia, pp. 124-133.

[10] R. Polikar, (2006) "Ensemble based systems in decision making- feature article", IEEE Circuits and Systems Magazine, Third quarter, pp. 21-45.

[11] P. V. Reddy, A. kumar, S. M. K. Rahman, and T. S. Mundra, (2007) "A new method for fingerprint antispoofing using pulse oximetry", Proceedings of the IEEE International Conference on Biometrics: Theory and Applications, Washington D. C., pp. 36-41.

[12] P. V. Reddy, A. kumar, S. M. K. Rahman, and T. S. Mundra, (2008), "A new antispoofing approach for biometric devices", IEEE Transactions on Biomedical Circuits and Systems, Vol. 2, No. 4, pp. 328-333.

[13] Y. S. Moon, J. S. Chen, K. C. Chan, K. So, and K. C. Woo, (2005), "Wavelet-based liveness detection", Electronics Letters, Vol. 41, No. 20, pp. 1112-1113.

[14] S. Parthasaradhi, R. Derakhshani, L. Hornak, S. A. C. Schuckers, (2005) "Time-series detection of perspiration as a liveness test in fingerprint scanners", IEEE Transactions on Systems, Man, and Cybernetics-Part C: Applications and Reviews, Vol. 35, No. 3, pp. 335-343.

[15] S. B. Nikam, S. Agarwal, (2008) "Local binary pattern and wavelet-based spoof fingerprint detection", International Journal of Biometrics, Vol. 1, No. 2, pp. 141-159. 
[16] M. Hong, C. S. Tong, (2006) "A fast and effective model for wavelet subband histograms and its application in texture image retrieval", IEEE Transactions on Image Processing, Vol. 15, No. 10, pp. 3078-3088.

[17] R. M. Haralick, K. Shamugam, and I. Dinstein, (1973) "Textural features for image classification", IEEE Transactions on Systems, Man, and Cybernetics, Vol. 3, No. 6, pp. 610-621.

[18] S. B. Nikam, S. Agarwal, (2008) "Fingerprint liveness detection using curvelet energy and cooccurrence signatures", Proceedings of $5^{\text {th }}$ International Conference on Computer Graphics, Visualization and Imaging, Penag, Malaysia, pp. 217-222.

[19] A. Abhyankar, S. Schuckers, (2006) "Fingerprint liveness detection using local ridge frequencies and multiresolution texture analysis techniques", Proceedings of International Conference on Image Processing, pp. 321-324.

\section{Authors}

Dr. Shankar Bhausaheb Nikam is a Lecturer in Department of Computer Engineering, Government Polytechnic, Awasari, Pune. He received the B.E. degree in Computer Science and Engineering from Government College of Engineering, Aurangabad in 1991, and M.E. degree in Computer Engineering from Government College of Engineering, Pune in 2001. He has completed Ph. D. in Computer Science and Engineering from Motilal Nehru National Institute of Technology, Allahabad in 2009. His research interests include Fingerprint- based biometrics.

Dr. Suneeta Agarwal is a Professor and Head of Computer Science and Engineering Department, Motilal Nehru National Institute of Technology, Allahabad, India. She received M. Sc., M. Tech. degrees and $\mathrm{Ph}$. D. from Indian Institute of Technology, Kanpur in 1980. She has worked on development of robotics project sponsored by Government of India. Her research interests include Automata Theory, Compression, Pattern matching, Fingerprint recognition.
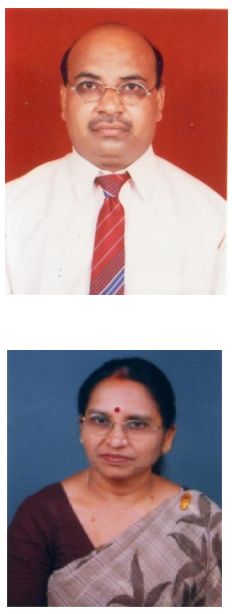